\title{
Impregnated Copper on Magnetite as a Heterogeneous Catalyst for the Homocoupling of Terminal Alkynes
}

\author{
Juana M. Pérez, Rafael Cano, Miguel Yus, Diego J. Ramón* \\ Departamento Química Orgánica and Instituto de Síntesis Orgánica, Universidad de Alicante, Apdo. 99, E-03080 Alicante, Spain \\ Fax: +0034-965903549 \\ E-mail: djramon@ua.es
}

Received: The date will be inserted once the manuscript is accepted.

\begin{abstract}
Impregnated copper on magnetite is a versatile heterogeneous system for the synthesis of 1,3-diynes by the homocoupling of terminal alkynes. This catalyst does not require the use of oxygen pressure as oxidant, solvent or harsh conditions to achieve the expected products. Moreover, the catalyst is very easy to remove from the reaction medium simply by using a magnet. The reaction is carried out with the lowest copper loading for a heterogeneous catalyst.
\end{abstract}

Key words: alkynes, copper, heterogeneous catalysis, iron, supported catalysis

The homocoupling of terminal alkynes ${ }^{1}$ to obtain 1,3diynes have attracted much attention in organic chemistry due to the role of diynes as building blocks in many natural products ${ }^{2}$ and pharmaceuticals with anti-inflammatory, antibacterial, antitumor and antifungal activities. Also they have caught the interest of chemists as interesting materials like precursors of polymers, ${ }^{3}$ macrocycles ${ }^{4}$ and supramolecular structures. ${ }^{5}$

The symmetric coupling of simple terminal acetylenes, ${ }^{6}$ named Glaser-Hay reaction, was discovered over a century and soon the methodology was improved. ${ }^{7}$ Among the different employed metallic salts, copper emerged as the best metal for this transformation. In fact, the number of different copper-complexes capable to perform successfully this transformation is continuously increasing. ${ }^{8}$ However, homogeneous catalysts have some important drawbacks, such as the required high metal loading and the no-recyclability of catalyst, amongst others. Only the homogeneous copper acetatepolyethyleneglycol catalyst system $^{86}$ has been recycled up to five-fold, although with a decrease in the activity after the reactivation step by acetic acid treatment.

Heterogeneous catalysts were designed to perform this transformation with an easy method for removal, recovering and recycling the catalysts. Although, there are several examples of insoluble copper derivatives able to perform the Glaser-Hay reaction, ${ }^{9}$ the most used strategy has been supporting copper salts on different inert oxides such as hydrotalcite, ${ }^{10}$ alumina, ${ }^{11}$ zeolite, $^{12}$ titania, ${ }^{13}$ a silicotungstate complex, ${ }^{14}$ molecular sieves ${ }^{15}$ and mesoporous silica. ${ }^{16}$ However, there are still some drawbacks that should be improved, such as the high metal catalyst loading required, ${ }^{9-16}$ the high temperatures used, ${ }^{12,13 a, 14,15}$ the use of non-environmentally benign solvents, $9 \mathrm{~b}, 10 \mathrm{~b}, \mathrm{c}, 12,13 \mathrm{a}, 14-16$ the need of oxygen pressure $^{10 a, b, 13 a, 14,15}$ and the recyclability of the catalyst in some cases. ${ }^{9 a, b, 10 b, 11,12,13 a}$

On the other hand, an interesting cooperative effect was observed when the reaction was carried out using homogenous copper and an iron salt. ${ }^{17}$ In this case, the copper salt loading could be reduced to $0.1 \mathrm{~mol} \%$ in the presence of $10 \mathrm{~mol} \%$ of iron(III) acetylacetonate. We have recently developed a new, simple and robust method to immobilize different metal oxides ${ }^{18}$ onto magnetite. ${ }^{19}$ With the aforementioned cooperative effect in mind, we decided to apply the copper impregnated on magnetite catalyst ${ }^{20}$ to the Glaser-Hay reaction. Along this research line, we report herein the first catalyst derived from the cheaper and safer firstrow copper transition metal impregnated on magnetite and its use in the coupling of terminal alkynes.

The homocoupling of ethynylbenzene (1a) was selected as the reaction model in order to optimize the conditions (Table 1) in the presence of pyperidine as base. Initially, the reaction was tested in different solvents (Table 1, entries 1-9), finding that the best result was obtained in absence of solvent. An increment of the temperature of the reaction did not improve the results (entry 10) and the reaction at room temperature only gave traces of product 2a (entry 11). The reaction fails in absence of base (entry 12). The effect of the base in the reaction was also studied using different amines (entries 13-15) and similar yields were obtained in only 4 hours with primary amines. However the corresponding amides were obtained as byproducts. Using other organic and inorganic bases (entries 16-20), lower yields were obtained. The product 2a was obtained in quantitative yield in the case of using $t$-BuOK, (entry 21). When the reaction was carried out using $50 \mathrm{~mol} \%$ of base the yield decreased (entry 22), so the conclusion was that stoichiometric amount of base was needed for the homocoupling (entries 21-22). An increment in the amount of base did not reduce the reaction time (entry 23). When the reaction was performed in argon atmosphere the yield decreased to 38\% (entry 24). This result confirmed that the oxygen present in the air played an important role in the process, being the final oxidant source. Then, the amount of catalyst employed was optimized (entries 25-27). A decrease of the catalyst amount to $0.26 \mathrm{~mol} \%$ produced a $99 \%$ 
yield of 2a, although longer reaction time (48 h) was necessary (entry 25). The reaction with a higher or lower loading of the metal did not improve the results (entries 26 and 27). Finally, the reaction under the best conditions was repeated in absence of catalyst and the starting material was recovered after a week (entry 28). It should be pointed out that the reaction under an oxygen atmosphere did not change the previous result (compare entries 21 and 29).

Table 1 Optimization of the reaction conditions. ${ }^{\mathrm{a}}$

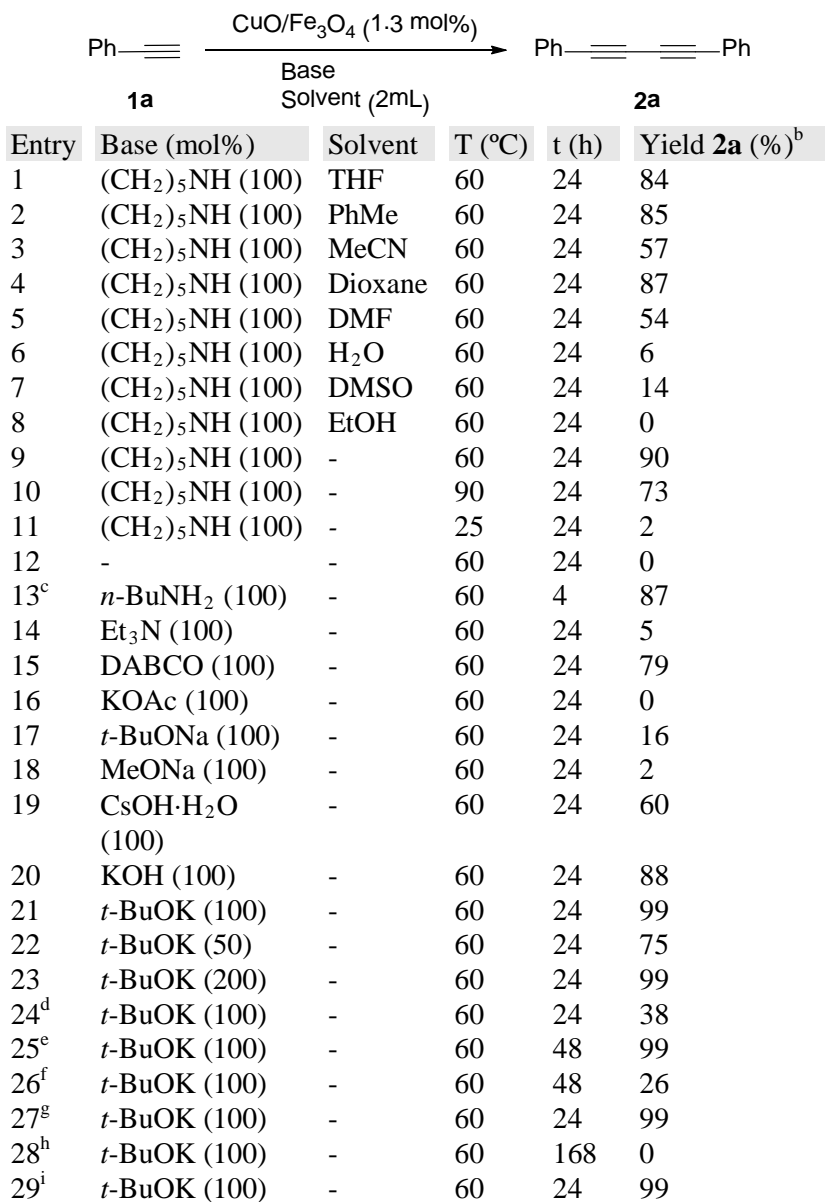

${ }^{a}$ Reaction carried out using 1a (2 mmol) under air atmosphere.

${ }^{\mathrm{b}}$ Isolated yields after column chromatography.

${ }^{c} N$-Butyl-2-phenylacetamide was isolated in $13 \%$ yield.

${ }^{\mathrm{d}}$ Reaction carried out under Ar atmosphere.

e Reaction carried out using $0.26 \mathrm{~mol} \%$ of catalyst.

${ }^{\mathrm{f}}$ Reaction carried out using $0.13 \mathrm{~mol} \%$ of catalyst.

${ }^{\mathrm{g}}$ Reaction carried out using $2.6 \mathrm{~mol} \%$ of catalyst.

${ }^{\mathrm{h}}$ Reaction carried out in absence of catalyst.

${ }^{1}$ Reaction carried out under $\mathrm{O}_{2}$ atmosphere.

Then, with the optimized conditions in hand, different catalysts, prepared by a simple impregnation protocol, were tested (Table 2). First of all, the activity of the support was evaluated, performing the reaction using only magnetite as catalyst. Nano and microparticles of magnetite were used, and the results showed that magnetite had less activity, needing longer reaction times and reaching lower yields (compare entries 1, 2 and 3). Ruthenium, ${ }^{18 \mathrm{~b}}$ cobalt, ${ }^{18 \mathrm{~b}}$ iridium, ${ }^{18 \mathrm{e}}$ palladium $^{18 \mathrm{c}}$ and platinum ${ }^{18 \mathrm{~d}}$ did not show any activity for this transformation. Nickel catalyst ${ }^{18 b}$ showed a moderate activity giving the expected product in $41 \%$ yield (entry 7). The reaction using a bimetallic catalyst was tried, obtaining the expected product in high yields (entries 10-11), with the copper-nickel ${ }^{18 c}$ catalyst reaching a quantitative yield. The reaction using only copper oxide or copper oxide together with magnetite gave a slightly lower yield than the impregnated catalyst one (compare entries 1, 12 and 13), but we do not have any clear explanation for this cooperative effect.

Table 2 Optimization of catalyst. ${ }^{\text {a }}$

\begin{tabular}{|c|c|c|c|}
\hline \multirow{2}{*}{$\begin{array}{c}\mathrm{Ph}_{-} \\
1 \mathrm{\equiv}\end{array}$} & catalyst & \multicolumn{2}{|c|}{$\mathrm{Ph}=\overline{P h}$} \\
\hline & $\begin{array}{l}t \text {-BuOK }(100 \mathrm{~mol} \%) \\
60^{\circ} \mathrm{C}\end{array}$ & \multicolumn{2}{|c|}{$-\quad-$} \\
\hline \multicolumn{2}{|c|}{ Catalyst (mol\%) } & $t(d)$ & Yield 2a (\%) \\
\hline \multicolumn{2}{|c|}{$\mathrm{CuO}-\mathrm{Fe}_{3} \mathrm{O}_{4}(0.26)$} & 2 & 99 \\
\hline \multicolumn{2}{|c|}{$\mathrm{Fe}_{3} \mathrm{O}_{4}(0.13)$} & 7 & 68 \\
\hline \multicolumn{2}{|c|}{$\mathrm{Fe}_{3} \mathrm{O}_{4}(0.13)^{\mathrm{C}}$} & 7 & 78 \\
\hline \multicolumn{2}{|c|}{$\mathrm{Ru}_{2} \mathrm{O}_{3}-\mathrm{Fe}_{3} \mathrm{O}_{4}(0.28)$} & 2 & 0 \\
\hline \multicolumn{2}{|c|}{$\mathrm{CoO}-\mathrm{Fe}_{3} \mathrm{O}_{4}(0.35)$} & 2 & 0 \\
\hline \multicolumn{2}{|c|}{$\mathrm{IrO}_{2}-\mathrm{Fe}_{3} \mathrm{O}_{4}(0.03)$} & 2 & 0 \\
\hline \multicolumn{2}{|c|}{$\mathrm{NiO}-\mathrm{Fe}_{3} \mathrm{O}_{4}(0.2)$} & 2 & 41 \\
\hline \multicolumn{2}{|c|}{$\mathrm{PdO}-\mathrm{Fe}_{3} \mathrm{O}_{4}(0.24)$} & 2 & 1 \\
\hline \multicolumn{2}{|c|}{$\mathrm{PtO} / \mathrm{PtO}_{2}-\mathrm{Fe}_{3} \mathrm{O}_{4}(0.12)$} & 2 & 1 \\
\hline \multicolumn{2}{|c|}{$\mathrm{Pd}(\mathrm{II}, 0) / \mathrm{Cu}(\mathrm{II})-\mathrm{Fe}_{3} \mathrm{O}_{4}(0.3 / 0.16)$} & 2 & 89 \\
\hline \multicolumn{2}{|c|}{$\mathrm{Cu}(\mathrm{II}, 0) / \mathrm{Ni}(\mathrm{II})-\mathrm{Fe}_{3} \mathrm{O}_{4}(0.22 / 0.18)$} & 2 & 99 \\
\hline \multicolumn{2}{|c|}{$\mathrm{CuO}(0.26)$} & 2 & 89 \\
\hline \multicolumn{2}{|c|}{$\mathrm{CuO}(0.26)+\mathrm{Fe}_{3} \mathrm{O}_{4}(0.13)$} & 2 & 88 \\
\hline
\end{tabular}

Once the optimal reaction conditions were established, the problem of recycling was examined (Fig. 1). The catalyst recovered by a magnet from the reaction was washed with methanol and re-used under the same reaction conditions, obtaining the expected product 2a in 99\% yield. However, in the fourth cycle, the yield was only $75 \%$, indicating that there is a small decrease in the activity of the catalyst and in the fifth cycle the yield dropped down to $20 \%$.

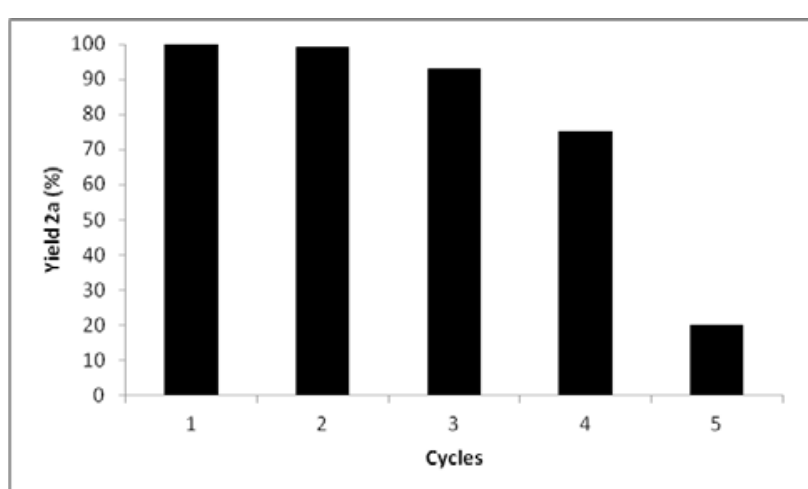

Figure 1 Yields obtained with a recycled $\mathrm{CuO}-\mathrm{Fe}_{3} \mathrm{O}_{4}$ catalyst 
The nano-size distribution of the catalyst obtained from TEM-images was the same after reaction, with the copper oxide particles at the surface of the catalyst being about $68 \%$ between 2 and $6 \mathrm{~nm}$ after reaction and $73 \%$ before the reaction. The XPS studies on the used catalyst showed the partial reduction of copper from the initial $4: 1$ mixture of $\mathrm{Cu}(\mathrm{II}: 0)$ to a $1: 1$ mixture. The ICP-MS analysis of the reaction solution showed the presence of copper $(6.2 \%$ of the initial amount) and iron (0.22\% of the initial amount), a similar result was obtained when the elimination of catalyst was performed without cooling down the mixture. These results seem to indicate that not all copper on the surface of magnetite has the same activity, and the most active species seems to leach faster.

Once the optimal reaction conditions were established the optimized protocol was applied to other substrates. The reaction using different substituted arylalkynes $\mathbf{1}$ gave the expected products 2 . The reactions seems to be more affected by the presence of chelating groups than for the electronic nature of them, with the reagents with strongest chelating ability giving the lowest yields (compare entries 1-5). The presence of a steric hindrance at the aromatic decreased the yield (entry 6). Finally, the reaction could also be performed using the less reactive aliphatic alkynes and the expected products 2 were obtained in all the tested cases (entries 10-14), albeit with slightly lower yields.

Table 3 Preparation of 1,3-diynes. ${ }^{\mathrm{a}}$

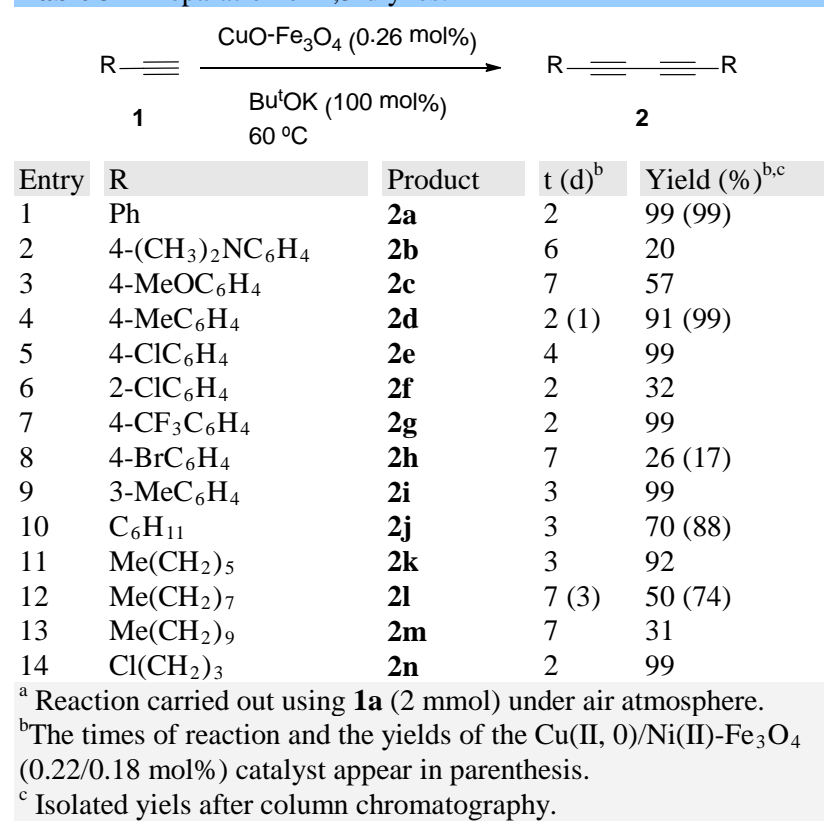

Encouraged by the success obtained in the homocoupling of terminal alkynes, the hydration reaction of 1,3-diynes to afford the corresponding 2,5disubstituted furans was examined, which is catalyzed by different copper salts. ${ }^{21}$ We performed the reaction with compound 2a in DMSO in presence of $\mathrm{KOH}$ and
$\mathrm{CuO}-\mathrm{Fe}_{3} \mathrm{O}_{4}$ at $80{ }^{\circ} \mathrm{C}$. To our delight the desired furan 3a was obtained in quantitative yield. However, it should be pointed out that the hydration reaction, in absence of catalyst, gave surprisingly furan 3a with the same yield. Then, a two-step one-pot process was tried, in order to the direct transformation of alkynes to the corresponding 2,5-disunstituted furans 3 (Table 4). After the corresponding diyne was obtained in the first step the catalyst was removed with a magnet, and without purification of the reagents, DMSO and aqueous $\mathrm{KOH}$ were added. Excellent results were obtained for all types of 4-substituted aryl diynes (entries 2-5). Unfortunately, when an aliphatic diyne was used the reaction failed (entry 5), recovering the corresponding diyne 2 .

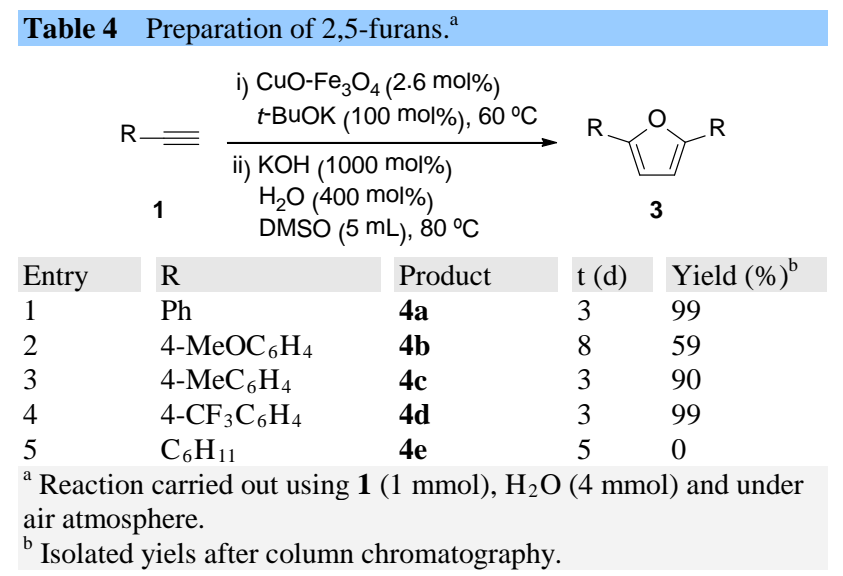

Finally, the decarboxylative coupling reaction ${ }^{22}$ of phenylpropiolic acid (4) was tested. Copper catalyst gave only a $21 \%$ of the expected product 2a. However the expected product was obtained with quantitative yield when $\mathrm{NiO}-\mathrm{Cu}-\mathrm{Fe}_{3} \mathrm{O}_{4}$ was used as catalyst (Scheme 1).

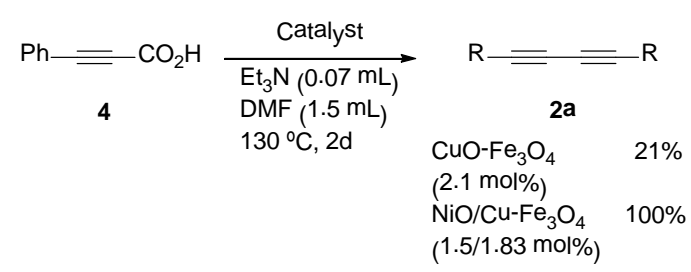

Scheme 1 Decarboxylative coupling reaction of phenylpropiolic acid

In conclusion, we have demonstrated that the impregnated copper on magnetite catalyst is a good catalyst for the homocoupling of terminal alkynes under solvent-free conditions, in absence of oxygen pressure and with the lowest loading of copper as described in the literature for a heterogeneous catalyst. Moreover, the catalyst is easily removed from the reaction media just by the use of a simple magnet and 
could be re-used up to three times without a detrimental effect on the yield.

Melting points were obtained with a Reichert Thermovar apparatus. NMR spectra were recorded on a Bruker AC-300 (300 MHz for ${ }^{1} \mathrm{H}$ and $75 \mathrm{MHz}$ for ${ }^{13} \mathrm{C}$ ) using $\mathrm{CDCl}_{3}$ as a solvent and TMS as internal standard for ${ }^{1} \mathrm{H}$ and ${ }^{13} \mathrm{C}$; chemical shifts are given in $\delta$ (parts per million) and coupling constants $(J)$ in Hertz. FT-IR spectra were obtained on a JASCO 4100LE (Pike Miracle ATR) spectrophotometer. Mass spectra (EI) were obtained at 70 $\mathrm{eV}$ on a Himazdu QP-5000 spectrometer, giving fragment ions in $\mathrm{m} / \mathrm{z}$ with relative intensities (\%) in parentheses. Thin layer chromatography (TLC) was carried out on Schleicher \& Schuell F1400/LS 254 plates coated with a $0.2 \mathrm{~mm}$ layer of silica gel; detection by $\mathrm{UV}_{254}$ light, staining with phosphomolybdic acid [25 g phosphomolybdic acid, $10 \mathrm{~g}$ $\mathrm{Ce}\left(\mathrm{SO}_{4}\right)_{2} \cdot 4 \mathrm{H}_{2} \mathrm{O}, 60 \mathrm{~mL}$ of concentrated $\mathrm{H}_{2} \mathrm{SO}_{4}$ and $940 \mathrm{ml}$ $\mathrm{H}_{2} \mathrm{O}$. The chromatographic analyses were determined with an instrument equipped with a flame ionization detector and a $30 \mathrm{~m}$ capillary column $(0.32 \mathrm{~mm}$ diam, $0.25 \mu \mathrm{m}$ film thickness, HP-5 stationary phase), using nitrogen $(2 \mathrm{~mL} / \mathrm{min})$ as carrier gas), $\mathrm{P}=10 \mathrm{psi}, \mathrm{T}_{\text {injector }}$ $=270{ }^{\circ} \mathrm{C}$ and $\mathrm{T}_{\text {detector }}=300{ }^{\circ} \mathrm{C} ; \mathrm{t}_{\mathrm{r}}$ values are given under these conditions. Column chromatography was performed using silica gel 60 of 35-70 mesh. All reagents were commercially available (Acros, Aldrich, Fluorochem) and were used as received. The ICP-MS analyses were carried out on a Thermo Elemental VGPOExCell spectrometer. The X-ray Fluorescence analyses were carried out on a PHILIPS MAGIX PRO (PW2400) X-ray spectrometer equipped with a rhodium $\mathrm{X}$-ray tube and a beryllium window. The reactions were performed in a 12vessel carrousel reaction station (Radleys). The $\mathrm{NiO} / \mathrm{Cu}-$ $\mathrm{Fe}_{3} \mathrm{O}_{4}$ and $\mathrm{CuO}-\mathrm{Fe}_{3} \mathrm{O}_{4}$ catalyst were prepared according to the literature procedure. ${ }^{18 \mathrm{c}, 20 \mathrm{c}}$.

\section{General procedure for the catalyst preparation.}

To a stirred solution of corresponding $\mathrm{MCl}_{\mathrm{n}}(1 \mathrm{mmol})$, or $\mathrm{MCl}_{\mathrm{n}}(1 \mathrm{mmol})$ and $\mathrm{M}^{\prime} \mathrm{Cl}_{\mathrm{n}}$, (1 mmol) for bimetallic species, in deionised water $(120 \mathrm{~mL})$ was added commercial available $\mathrm{Fe}_{3} \mathrm{O}_{4}$ (4 g, $17 \mathrm{mmol}$, powder $<5 \mu \mathrm{m}$, BET area: $9.86 \mathrm{~m}^{2} / \mathrm{g}$ ). In the case of palladium and copper-palladium catalyst, a great excess of potassium chloride ( $13 \mathrm{mmol}, 1 \mathrm{~g}$ ) was added also to the initial solution in order to increase the solubility of the palladium chloride. After 10 minutes at room temperature, the mixture was slowly basified with $\mathrm{NaOH}(1 \mathrm{M})$ until $\mathrm{pH}$ around 13. The mixture was stirred during one day at room temperature in air. After that, the catalyst was filtered and washed several times with deionised water $(3 \times 10 \mathrm{~mL})$. The solid was dried at $100{ }^{\circ} \mathrm{C}$ during $24 \mathrm{~h}$ in a standard glassware oven obtaining the expected catalyst.

\section{General procedure for the synthesis of 1,3-diynes 2.}

To a stirred solution of the corresponding alkyne (1, 2 mmol) were added $\mathrm{CuO}-\mathrm{Fe}_{3} \mathrm{O}_{4}(10 \mathrm{mg}, 0.26 \mathrm{~mol} \%)$ or $\mathrm{NiO} / \mathrm{Cu}-\mathrm{Fe}_{3} \mathrm{O}_{4}$ (10 mg, 0.18/0.22 mol\%) and $t$-BuOK (224 mg, $2 \mathrm{mmol}$ ) under air atmosphere. The corresponding mixture was vigorous stirred at $60{ }^{\circ} \mathrm{C}$ until the end of the reaction. The catalyst was removed by a magnet and washed with EtOAc (2 $\times 5 \mathrm{~mL})$ and $\mathrm{H}_{2} \mathrm{O}(2 \times 5 \mathrm{~mL})$. The collected organic phases were dried over $\mathrm{MgSO}_{4}$, and the solvents were removed under reduced pressure. The product was purified by column chromatography to give the corresponding pure products 2 . Yields, physical and spectroscopic data, as well as literature data for known compounds, follow:

\section{1,4-Diphenylbuta-1,3-diyne (2a) ${ }^{13 b}$}

White solid; yield: $200 \mathrm{mg}(99 \%) ; \mathrm{t}_{\mathrm{r}}=15.8 \mathrm{~min}$ [ $60{ }^{\circ} \mathrm{C}$ (3 min)$\left.15{ }^{\circ} \mathrm{C} / \mathrm{min}-270{ }^{\circ} \mathrm{C}(15 \mathrm{~min})\right]$; $\mathrm{Mp}=83-85^{\circ} \mathrm{C}$ (hexane); $\mathrm{R}_{\mathrm{f}}=$ 0.67 (Hexane-EtOAc, 4:1).

IR (ATR): 3050, 1593, $1485 \mathrm{~cm}^{-1}$.

${ }^{1} \mathrm{H}$ NMR (300MHz, $\mathrm{CDCl}_{3}$ ): $\delta=7.25-7.3(\mathrm{~m}, 6 \mathrm{H}, \mathrm{ArH}), 7.45-$ 7.5 (m, 4H, ArH).

${ }^{13} \mathrm{C}$ NMR (75 MHz, $\mathrm{CDCl}_{3}$ ): $\delta=73.9$ (2C), 81.5 (2C), 121.8 (2C), 128.4 (4C), 129.2 (2C), 132.5 (4C).

MS (EI, $70 \mathrm{eV}): m / z(\%)=101$ (8), 200 (22), 201 (11), 202 (100) $[\mathrm{M}]^{+}, 203(17)[\mathrm{M}+\mathrm{H}]^{+}$.

\section{4,4'-(Buta-1,3-diyne-1,4-diyl)bis( $N, N$-dimethylaniline) (2b) ${ }^{9 \mathrm{a}}$}

Pale brown solid; yield: $58 \mathrm{mg}(20 \%) ; \mathrm{t}_{\mathrm{r}}=13.4 \mathrm{~min}\left[60^{\circ} \mathrm{C}(3\right.$ min)- $\left.15{ }^{\circ} \mathrm{C} / \min -270{ }^{\circ} \mathrm{C}(15 \mathrm{~min})\right]$; $\mathrm{Mp}=215-217^{\circ} \mathrm{C}$ (hexane); $\mathrm{R}_{\mathrm{f}}=0.43$ (Hexane-EtOAc, 4:1).

IR (ATR): 1598, 1358, $1225 \mathrm{~cm}^{-1}$.

${ }^{1} \mathrm{H}$ NMR $\left(300 \mathrm{MHz}, \mathrm{CDCl}_{3}\right): \delta=3.0\left(\mathrm{~s}, 12 \mathrm{H}, \mathrm{CH}_{3}\right.$ x 4), 6.656.7 (d, $J=8.8 \mathrm{~Hz}, 4 \mathrm{H}, \mathrm{ArH}), 7.35-7.4$ (d, $J=8.8 \mathrm{~Hz}, 4 \mathrm{H}, \mathrm{ArH}$ ).

${ }^{13} \mathrm{C}$ NMR (75 MHz, $\left.\mathrm{CDCl}_{3}\right): \delta=40.2$ (4C), 74.7 (2C), 84.8 (2C), 108.8 (2C), 111.7 (4C), 133.2 (4C), 150.4 (2C).

MS (EI, $70 \mathrm{eV}): m / z(\%)=51$ (15), 77 (40), 78 (11), 155 (19), 183 (12), 201 (70), 202 (100), 203 (22), 207 (60), 208 (14), 229 (19), 269 (10), $288(10)[\mathrm{M}]^{+}$.

\section{1,4-Bis(4-methoxyphenyl)buta-1,3-diyne (2c) ${ }^{23}$}

Pale yellow solid; yield: $150 \mathrm{mg}(57 \%) ; \mathrm{t}_{\mathrm{r}}=20.7 \mathrm{~min}\left[60^{\circ} \mathrm{C}(3\right.$ $\min )-15{ }^{\circ} \mathrm{C} / \mathrm{min}-270{ }^{\circ} \mathrm{C}$ (15 min)]; $\mathrm{Mp}=131-133^{\circ} \mathrm{C}$ (hexane); $\mathrm{R}_{\mathrm{f}}=0.37$ (Hexane-EtOAc, 4:1).

IR (ATR): 3004, 2939, 2837, 1598, $1502 \mathrm{~cm}^{-1}$.

${ }^{1} \mathrm{H} \mathrm{NMR}\left(300 \mathrm{MHz}, \mathrm{CDCl}_{3}\right): \delta=3.82\left(\mathrm{~s}, 6 \mathrm{H}, \mathrm{CH}_{3} \times 2\right)$, 6.86.85 (d, $J=8.8 \mathrm{~Hz}, 4 \mathrm{H}, \mathrm{ArH}), 7.45-7.5$ (d, $J=8.8 \mathrm{~Hz}, 4 \mathrm{H}$, $\mathrm{ArH})$.

${ }^{13} \mathrm{C}$ NMR (75 MHz, $\mathrm{CDCl}_{3}$ ): $\delta=55.3$ (2C), 72.9 (2C), 81.2 (2C), 113.9 (2C), 114.1 (4C), 134.0 (4C), 160.2 (2C).

MS (EI, $70 \mathrm{eV}): \mathrm{m} / \mathrm{z}(\%)=131$ (13), 176 (15), 219 (14), 247 (58), 248 (11), $262(100)[\mathrm{M}]^{+}, 263(19)[\mathrm{M}+\mathrm{H}]^{+}$. 


\section{1,4-Di-p-tolylbuta-1,3-diyne (2d) ${ }^{15}$}

Pale yellow solid; yield: $209 \mathrm{mg}(91 \%) ; \mathrm{t}_{\mathrm{r}}=17.5 \mathrm{~min}\left[60^{\circ} \mathrm{C}(3\right.$ min)- $\left.15{ }^{\circ} \mathrm{C} / \mathrm{min}-270{ }^{\circ} \mathrm{C}(15 \mathrm{~min})\right] ; \mathrm{Mp}=118-120^{\circ} \mathrm{C}$ (hexane); $\mathrm{R}_{\mathrm{f}}=0.8$ (Hexane-EtOAc, 4:1).

IR (ATR): 3032, $1501 \mathrm{~cm}^{-1}$.

${ }^{1} \mathrm{H}$ NMR $\left(300 \mathrm{MHz}, \mathrm{CDCl}_{3}\right): \delta=2.34$ (s, 6H, $\mathrm{CH}_{3} \times 2$ ), 7.1$7.15(\mathrm{~d}, J=8.0 \mathrm{~Hz}, 4 \mathrm{H}, \mathrm{ArH}), 7.35-7.4(\mathrm{~d}, J=8.0 \mathrm{~Hz}, 4 \mathrm{H}$, $\mathrm{ArH})$.

${ }^{13} \mathrm{C}$ NMR (75 MHz, $\mathrm{CDCl}_{3}$ ): $\delta=21.6$ (2C), 73.4 (2C), 81.5 (2C), 118.8 (2C), 129.2 (4C), 132.4 (4C), 139.5 (2C).

MS (EI, $70 \mathrm{eV}): m / z(\%)=215$ (17), 226 (13), 228 (12), 229 (22), $230(100)[\mathrm{M}]^{+}, 231(19)[\mathrm{M}+\mathrm{H}]^{+}$.

\section{1,4-Bis(4-chlorophenyl)buta-1,3-diyne (2e) $)^{15}$}

Pale yellow solid; yield: $268 \mathrm{mg}(99 \%) ; \mathrm{t}_{\mathrm{r}}=18.0 \mathrm{~min}\left[60^{\circ} \mathrm{C}(3\right.$ $\left.\min )-15{ }^{\circ} \mathrm{C} / \min -270{ }^{\circ} \mathrm{C}(15 \mathrm{~min})\right] ; \mathrm{Mp}=165-167{ }^{\circ} \mathrm{C}$ (hexane); $\mathrm{R}_{\mathrm{f}}=0.77$ (Hexane-EtOAc, 4:1).

IR (ATR): 1483, 1395, $1092 \mathrm{~cm}^{-1}$.

${ }^{1} \mathrm{H}$ NMR $\left(300 \mathrm{MHz}, \mathrm{CDCl}_{3}\right): \delta=7.3-7.35(\mathrm{~d}, J=8.7 \mathrm{~Hz}, 4 \mathrm{H}$, ArH), 7.45-7.5 (d, $J=8.7 \mathrm{~Hz}, 4 \mathrm{H}, \mathrm{ArH})$.

${ }^{13} \mathrm{C}$ NMR (75 MHz, $\mathrm{CDCl}_{3}$ ): $\delta=76.6$ (2C), 80.8 (2C), 119.3 (2C), 128.9 (4C), 133.7 (4C), 134.0 (2C).

MS (EI, $70 \mathrm{eV}): \mathrm{m} / \mathrm{z}(\%)=200(28), 270(100)[\mathrm{M}]^{+}, 271(18)$ $[\mathrm{M}+\mathrm{H}]^{+}, 272(65)[\mathrm{M}+2]^{+}, 273(11),[\mathrm{M}+3]^{+}, 274$ (11), [M $+4]^{+}$.

\section{1,4-Bis(2-chlorophenyl)buta-1,3-diyne (2f) ${ }^{9 \mathrm{c}}$}

White solid; yield: $87 \mathrm{mg}(32 \%) ; \mathrm{t}_{\mathrm{r}}=18.4 \mathrm{~min}\left[60^{\circ} \mathrm{C}\right.$ (3 min)$15{ }^{\circ} \mathrm{C} / \mathrm{min}-270{ }^{\circ} \mathrm{C}$ (15 min)]; $\mathrm{Mp}=135-138{ }^{\circ} \mathrm{C}$ (hexane); $\mathrm{R}_{\mathrm{f}}=$ 0.5 (Hexane-EtOAc 4:1).

IR (ATR): 3068, 1463, 1433, $1053 \mathrm{~cm}^{-1}$.

${ }^{1} \mathrm{H}$ NMR $\left(300 \mathrm{MHz}, \mathrm{CDCl}_{3}\right): \delta=7.2-7.25\left(\mathrm{td}, J=7.5 \mathrm{~Hz},{ }^{4} J=\right.$ $1.2 \mathrm{~Hz}, 2 \mathrm{H}, \mathrm{ArH}), 7.3-7.35$ (td, $J=7.5 \mathrm{~Hz},{ }^{4} J=1.7 \mathrm{~Hz}, 2 \mathrm{H}$, ArH), 7.4-7.45 (dd, $\left.J=8.0 \mathrm{~Hz},{ }^{4} J=1.2 \mathrm{~Hz}, 2 \mathrm{H}, \mathrm{ArH}\right), 7.55-7.6$ (dd, $J=7.6 \mathrm{~Hz},{ }^{4} J=1.7 \mathrm{~Hz}, 2 \mathrm{H}, \mathrm{ArH}$ ).

${ }^{13} \mathrm{C}$ NMR (75 MHz, $\mathrm{CDCl}_{3}$ ): $\delta=78.3$ (2C), 79.4 (2C), 121.8 (2C), 126.5 (2C), 129.4 (2C), 130.3 (2C), 134.4 (2C), 136.9 (2C).

MS (EI, $70 \mathrm{eV}): m / z(\%)=200$ (34), $270(100)[\mathrm{M}]^{+}, 271$ (18) $[\mathrm{M}+\mathrm{H}]^{+}, 272(64)[\mathrm{M}+2]^{+}, 273(12),[\mathrm{M}+3]^{+}, 274$ (12), [M $+4]^{+}$.

\section{1,4-Bis((4-trifluoromethyl)phenyl)buta-1,3-diyne (2g) ${ }^{24}$}

Pale yellow solid; yield: $335 \mathrm{mg}(99 \%) ; \mathrm{t}_{\mathrm{r}}=15.0 \mathrm{~min}\left[60^{\circ} \mathrm{C}(3\right.$ $\min )-15{ }^{\circ} \mathrm{C} / \mathrm{min}-270{ }^{\circ} \mathrm{C}$ (15 min)]; $\mathrm{Mp}=165-168{ }^{\circ} \mathrm{C}$ (hexane); $\mathrm{R}_{\mathrm{f}}=0.7$ (Hexane-EtOAc, 4:1).

IR (ATR): 1610, $1407 \mathrm{~cm}^{-1}$.

${ }^{1} \mathrm{H}$ NMR (300MHz, $\mathrm{CDCl}_{3}$ ): $\delta=7.6-7.65$ (m, 8H, ArH).

${ }^{13} \mathrm{C}$ NMR (75 MHz, $\mathrm{CDCl}_{3}$ ): $\delta=75.6(2 \mathrm{C}), 80.9$ (2C), 123.7 (2C, q, $\left.{ }^{1} J_{(C-F)}=272.4 \mathrm{~Hz}\right), 125.3(2 \mathrm{C}), 125.4\left(4 \mathrm{C}, \mathrm{q},{ }^{3} \mathrm{~J}_{(\mathrm{C}-F)}=\right.$ $3.8 \mathrm{~Hz}), 131.1\left(2 \mathrm{C}, \mathrm{q},{ }^{2} J_{(C-F)}=33 \mathrm{~Hz}\right), 132.8(4 \mathrm{C})$.
MS (EI, $70 \mathrm{eV}): m / z(\%)=319$ (17), $338(100)[\mathrm{M}]^{+}, 339$ (22) $[\mathrm{M}+\mathrm{H}]^{+}$.

\section{1,4-Bis(4-bromophenyl)buta-1,3-diyne (2h) ${ }^{25}$}

Pale yellow solid; yield: $94 \mathrm{mg}(26 \%) ; \mathrm{t}_{\mathrm{r}}=20.7 \mathrm{~min}\left[60^{\circ} \mathrm{C}(3\right.$ min)-15 $\left.{ }^{\circ} \mathrm{C} / \mathrm{min}-270{ }^{\circ} \mathrm{C}(15 \mathrm{~min})\right] ; \mathrm{Mp}=140-141^{\circ} \mathrm{C}$ (hexane); $\mathrm{R}_{\mathrm{f}}=0.73$ (Hexane-EtOAc, 4:1).

IR (ATR): 1480, $1066 \mathrm{~cm}^{-1}$.

${ }^{1} \mathrm{H} \mathrm{NMR}\left(300 \mathrm{MHz}, \mathrm{CDCl}_{3}\right): \delta=7.35-7.4(\mathrm{~d}, J=8.5 \mathrm{~Hz}, 4 \mathrm{H}$, ArH), 7.45-7.5 (d, $J=8.5 \mathrm{~Hz}, 4 \mathrm{H}, \mathrm{ArH})$.

${ }^{13} \mathrm{C}$ NMR (75 MHz, $\mathrm{CDCl}_{3}$ ): $\delta=77.2$ (2C), 81.0 (2C), 120.6 (2C), 131.7 (2C), 131.8 (4C), 133.8 (4C).

MS (EI, $70 \mathrm{eV}): m / z(\%)=174$ (10), 199 (11), 200 (34), 207 (19), 281 (12), 358 (52), 360 (100) [M] $]^{+}, 361$ (18) [M + H] .

\section{1,4-Bi-m-tolylbuta-1,3-diyne (2i) ${ }^{15}$}

Pale yellow solid; yield: $228 \mathrm{mg}(99 \%) ; \mathrm{t}_{\mathrm{r}}=17.3 \mathrm{~min}\left[60{ }^{\circ} \mathrm{C}(3\right.$ $\left.\min )-15^{\circ} \mathrm{C} / \mathrm{min}-270^{\circ} \mathrm{C}(15 \mathrm{~min})\right] ; \mathrm{Mp}=65-67^{\circ} \mathrm{C}$ (hexane); $\mathrm{R}_{\mathrm{f}}$ $=0.67$ (Hexane-EtOAc 4:1).

IR (film): 3035, $1479 \mathrm{~cm}^{-1}$.

${ }^{1} \mathrm{H}$ NMR $\left(300 \mathrm{MHz}, \mathrm{CDCl}_{3}\right): \delta=2.33$ (s, 6H, $\mathrm{CH}_{3} \times 2$ ), 7.157.25 (m, 4H, ArH), 7.3-7.35 (m, 4H, ArH).

${ }^{13} \mathrm{C}$ NMR (75 MHz, $\mathrm{CDCl}_{3}$ ): $\delta=\delta 21.1$ (2C), 73.6 (2C), 81.6 (2C), 121.6 (2C), 128.3 (2C), 129.6 (2C), 130.1 (2C), 132.9 (2C), 138.1 (2C).

MS (EI, $70 \mathrm{eV}): m / z(\%)=228$ (10), 229 (10), 230 (100) $[\mathrm{M}]^{+}$, $231(19)[\mathrm{M}+\mathrm{H}]^{+}$.

\section{1,4-Dicyclohexylbuta-1,3-diyne (2j) ${ }^{13 b}$}

Pale yellow solid; yield: $150 \mathrm{mg}(70 \%) ; \mathrm{t}_{\mathrm{r}}=15.1 \mathrm{~min}\left(60^{\circ} \mathrm{C}\right.$ (3min) $-15^{\circ} \mathrm{C} / \mathrm{min}-270^{\circ} \mathrm{C}(15 \mathrm{~min}) ; \mathrm{Mp}=77-82^{\circ} \mathrm{C}$ (hexane); $\mathrm{R}_{\mathrm{f}}=$ 0.97 (Hexane-EtOAc 4:1).

IR (film): 2925, 2852, $1447 \mathrm{~cm}^{-1}$.

${ }^{1} \mathrm{H}$ NMR (300MHz, $\left.\mathrm{CDCl}_{3}\right): \delta=1.15-1.25\left(\mathrm{~m}, 6 \mathrm{H}, \mathrm{CH}_{2} \times 3\right)$, 1.35-1.45 (m, 6H, $\left.\mathrm{CH}_{2} \times 3\right), 1.6-1.75$ (m, 8H, $\left.\mathrm{CH}_{2} \times 4\right)$, 2.352.4 (m, 2H, CH x 2).

${ }^{13} \mathrm{C}$ NMR (75 MHz, $\mathrm{CDCl}_{3}$ ): $\delta=24.8$ (4C), 25.7 (2C), 29.5 (2C), 32.3 (4C), 65.1 (2C), 81.9 (2C).

MS (EI, $70 \mathrm{eV}): \mathrm{m} / \mathrm{z}(\%)=51$ (13), 53 (13), 55 (23), 63 (18), 65 (17), 67 (39), 75 (10), 76 (14), 77 (33), 78 (17), 79 (43), 80 (26), 89 (15), 91 (100), 92 (23), 93 (15), 95 (17), 102 (11), 103 (16), 104 (32), 105 (33), 115 (55), 116 (16), 117 (82), 118 (28), 119 (17), 127 (14), 128 (47), 129 (63), 130 (20), 131 (62), 132 (15), 133 (11), 141 (14), 143 (39), 144 (14), 145 (27), 157 (21), 158 (11), 171 (35), 185 (16), 207 (18), 214 (84) [M] $]^{+}, 215$ (15) $[\mathrm{M}+\mathrm{H}]^{+}$.

\section{Hexadeca-7,9-diyne (2k) ${ }^{23}$}

Colorless oil; yield: $201 \mathrm{mg}(92 \%) ; \mathrm{t}_{\mathrm{r}}=14.1 \mathrm{~min}\left(60^{\circ} \mathrm{C}(3 \mathrm{~min})-\right.$ $15^{\circ} \mathrm{C} / \mathrm{min}-270^{\circ} \mathrm{C}(15 \mathrm{~min}) ; \mathrm{R}_{\mathrm{f}}=0.87$ (Hexane-EtOAc 4:1).

IR (ATR): 1465, 1459, 1378, $724 \mathrm{~cm}^{-1}$. 
${ }^{1} \mathrm{H}$ NMR (300MHz, $\left.\mathrm{CDCl}_{3}\right): \delta=0.85-0.95\left(\mathrm{~m}, 6 \mathrm{H}, \mathrm{CH}_{3} \times 2\right)$, 1.25-1.45 (m, $12 \mathrm{H}, \mathrm{CH}_{2} \times 6$ ), 1.45-1.55 (m, $\left.4 \mathrm{H}, \mathrm{CH}_{2} \times 2\right)$, 2.2$2.25\left(\mathrm{t}, J=6.8 \mathrm{~Hz}, 4 \mathrm{H}, \mathrm{CH}_{2} \times 2\right)$.

${ }^{13} \mathrm{C}$ NMR (75 MHz, $\mathrm{CDCl}_{3}$ ): $\delta=14.0$ (2C), 19.2 (2C), 22.5 (2C), 28.3 (2C), 28.5 (2C), 31.3 (2C), 65.2 (2C), 77.5 (2C).

MS (EI, $70 \mathrm{eV}): m / z(\%)=51$ (13), 55 (37), 63 (11), 65 (17), 67 (46), 69 (14), 76 (10), 77 (37), 78 (31), 79 (56), 80 (12), 81 (41), 91 (100), 92 (20), 93 (43), 95 (26), 105 (63), 106 (12), 107 (26), 117 (15), 119 (43), 121 (12), 133 (25), 147 (16), 161 (10), $218(0.3)[\mathrm{M}]^{+}$.

\section{Icosa-9,11-diyne (2I) ${ }^{23}$}

Colorless oil; yield: $137 \mathrm{mg}(50 \%) ; \mathrm{t}_{\mathrm{r}}=16.6 \mathrm{~min}\left(60^{\circ} \mathrm{C}(3 \mathrm{~min})-\right.$ $15^{\circ} \mathrm{C} / \mathrm{min}-270^{\circ} \mathrm{C}$ (15 min); $\mathrm{R}_{\mathrm{f}}=0.8$ (Hexane-EtOAc, 4:1).

IR (ATR): 2924, 2854, 1464, $722 \mathrm{~cm}^{-1}$.

${ }^{1} \mathrm{H}$ NMR (300MHz, $\left.\mathrm{CDCl}_{3}\right): \delta=0.85-0.9(\mathrm{t}, J=6.5 \mathrm{~Hz}, 6 \mathrm{H}$, $\left.\mathrm{CH}_{3} \times 2\right), 1.25-1.4\left(\mathrm{~m}, 20 \mathrm{H}, \mathrm{CH}_{2} \times 10\right), 1.45-1.55(\mathrm{~m}, 4 \mathrm{H}$, $\left.\mathrm{CH}_{2} \times 2\right), 2.2-2.25$ (t, $\left.J=6.9 \mathrm{~Hz}, 4 \mathrm{H}, \mathrm{CH}_{2} \times 2\right)$.

${ }^{13} \mathrm{C}$ NMR (75 MHz, $\mathrm{CDCl}_{3}$ ): $\delta=14.1$ (2C), 19.2 (2C), 22.6 (2C), 28.4 (2C), 28.8 (2C), 29.0 (2C), 29.1 (2C), 31.8 (2C), 65.2 (2C), 77.5 (2C).

MS (EI, $70 \mathrm{eV}): \mathrm{m} / \mathrm{z}(\%)=55$ (38), 57 (14), 65 (13), 67 (50), 69 (19), 77 (32), 78 (25), 79 (61), 80 (15), 81 (57), 82 (12), 91 (100), 92 (22), 93 (52), 94 (17), 95 (38), 103 (12), 105 (60), 106 (14), 107 (41), 108 (10), 109 (15), 115 (13), 117 (21), 119 (55), 120 (15), 121 (41), 131 (10), 133 (45), 134 (12), 135 (20), 147 (32), 148 (10), 149 (12), 161 (25), 175 (14), 274 (0) [M] ${ }^{+}$.

\section{Tetracosa-11,13-diyne (2m) $)^{26}$}

Pale yellow oil; yield: $102 \mathrm{mg}(31 \%) ; \mathrm{t}_{\mathrm{r}}=19.5 \mathrm{~min}\left[60^{\circ} \mathrm{C}(3\right.$ $\left.\min )-15{ }^{\circ} \mathrm{C} / \mathrm{min}-270{ }^{\circ} \mathrm{C}(15 \mathrm{~min})\right] ; \mathrm{R}_{\mathrm{f}}=0.8$ (Hexane-EtOAc, 4:1).

IR (ATR): 2953, 2923, 2853, 1464, $721 \mathrm{~cm}^{-1}$.

${ }^{1} \mathrm{H} \mathrm{NMR}\left(300 \mathrm{MHz}, \mathrm{CDCl}_{3}\right): \delta=0.85-0.9\left(\mathrm{~m}, 6 \mathrm{H}, \mathrm{CH}_{3} \times 2\right)$, 1.25-1.3 (m, 28H, $\left.\mathrm{CH}_{2} \times 14\right), 1.45-1.55$ (m, $4 \mathrm{H}, \mathrm{CH}_{2} \times 2$ ), 2.2$2.25\left(\mathrm{t}, J=7.1 \mathrm{~Hz}, 4 \mathrm{H}, \mathrm{CH}_{2} \times 2\right)$.

${ }^{13} \mathrm{C}$ NMR (75 MHz, $\mathrm{CDCl}_{3}$ ): $\delta=14.1$ (2C), 19.2 (2C), 22.7 (2C), 28.3 (2C), 28.8 (2C), 29.1 (2C), 29.3 (2C), 29.5 (2C), 29.6 (2C), 31.9 (2C), 65.2 (2C), 77.5 (2C).

MS (EI, $70 \mathrm{eV}): m / z(\%)=55$ (50), 57 (27), 65 (11), 67 (65), 69 (33), 77 (27), 78 (24), 79 (66), 80 (21), 81 (71), 82 (20), 83 (20), 91 (100), 92 (25), 93 (55), 94 (24), 95 (57), 96 (12), 103 (11), 105 (59), 106 (17), 107 (48), 108 (15), 109 (25), 115 (12), 117 (25), 119 (60), 120 (19), 121 (67), 122 (16), 123 (11), 131 (14), 133 (54), 134 (21), 135 (30), 147 (38), 148 (17), 149 (15), 161 (35), 162 (11), 163 (11), 175 (21), 189 (15), 330 (0) [M] $]^{+}$.

\section{1,10-Dichlorodeca-4,6-diyne (2n) ${ }^{23}$}

Pale yellow oil; yield: $200 \mathrm{mg}$ (99\%); $\mathrm{t}_{\mathrm{r}}=13.5 \mathrm{~min}\left[60^{\circ} \mathrm{C}(3\right.$ $\left.\min )-15{ }^{\circ} \mathrm{C} / \mathrm{min}-270{ }^{\circ} \mathrm{C}(15 \mathrm{~min})\right] ; \mathrm{R}_{\mathrm{f}}=0.67$ (Hexane-EtOAc, 4:1).

IR (ATR): 2960, 2927, $1288 \mathrm{~cm}^{-1}$.
${ }^{1} \mathrm{H}$ NMR (300MHz, $\mathrm{CDCl}_{3}$ ): $\delta=1.95-2.0$ (p, $J=6.5 \mathrm{~Hz}, 4 \mathrm{H}$, Cl- $\mathrm{CH}_{2} \mathrm{CH}_{2}$ ), 2.45-2.5 (t, $J=6.8 \mathrm{~Hz}, 4 \mathrm{H}, \mathrm{ClCH}_{2} \mathrm{CH}_{2} \mathrm{CH}_{2}$ ), 3.6-3.65 (t, $J=6.3 \mathrm{~Hz}, 4 \mathrm{H}, \mathrm{ClCH}_{2}$ ).

${ }^{13} \mathrm{C}$ NMR (75 MHz, $\mathrm{CDCl}_{3}$ ): $\delta=16.6$ (2C), 31.0 (2C), 43.4 (2C), 66.0 (2C), 75.8 (2C).

MS (EI, $70 \mathrm{eV}): m / z(\%)=51$ (29), 53 (11), 62 (10), 63 (36), 64 (12), 65 (22), 74 (16), 76 (25), 77 (86), 78 (25), 79 (12), 89 (29), 91 (100), 102 (14), 103 (93), 104 (33), 105 (33), 111 (11), 112 (26), 114 (61), 116 (38), 117 (45), 125 (47), 127 (20), 128 (10), 129 (20), 130 (10), 131 (39), 139 (42), 141 (15), 167 (20), 174 (20), 176 (16), 202 (88) [M] , 204 (61) [M + 2] , 206 (10) $[\mathrm{M}+4]^{+}$.

\section{General procedure for synthesis of 2,5-furans 3.}

To a stirred solution of the corresponding alkyne (1, 2 mmol) were added $\mathrm{CuO}-\mathrm{Fe}_{3} \mathrm{O}_{4}(10 \mathrm{mg}, 0.26 \mathrm{~mol} \%)$ and $t$ BuOK (112 mg, $1 \mathrm{mmol}$ ). The corresponding mixture was stirred at $60{ }^{\circ} \mathrm{C}$ until the end of the reaction 2-7 days, see Table $3)$. Then, the catalyst was removed by a magnet and washed with DMSO (5 mL). To the resulting solution of previous reaction residue and the aforementioned DMSO was added $\mathrm{KOH}$ (280 mg, $5 \mathrm{mmol}, 250 \mathrm{~mol} \%$ ) and $\mathrm{H}_{2} \mathrm{O}$ (4 mmol, 400 mol\%) in air atmosphere. The resulting solution was stirred at $80{ }^{\circ} \mathrm{C}$ during 1 day and quenched by addition of $\mathrm{H}_{2} \mathrm{O}(5 \mathrm{~mL})$. Then, the mixture was extracted with EtOAc $(2 \times 5 \mathrm{~mL})$ and the collected organic phases were dried over $\mathrm{MgSO}_{4}$. The solvent was removed under reduced pressure. The product was purified by column chromatography to give the corresponding pure product 3. Yields, physical and spectroscopic data, as well as literature data for known compounds, follow:

\section{2,5-Diphenylfuran (3a) ${ }^{21}$}

Pale yellow solid; yield: $109 \mathrm{mg}(99 \%) ; \mathrm{t}_{\mathrm{r}}=15.8 \mathrm{~min}\left[60^{\circ} \mathrm{C}\right.$ (3min)- $\left.15{ }^{\circ} \mathrm{C} / \mathrm{min}-270{ }^{\circ} \mathrm{C}(15 \mathrm{~min})\right] ; \mathrm{Mp}=47-52{ }^{\circ} \mathrm{C}$ (hexane); $\mathrm{R}_{\mathrm{f}}=0.73$ (Hexane-EtOAc, 4:1).

IR (ATR): 3022, 1259, 1023, $927 \mathrm{~cm}^{-1}$.

${ }^{1} \mathrm{H}$ NMR (300MHz, $\mathrm{CDCl}_{3}$ ): $\delta=6.75$ (s, 2H, CH x 2), 7.25-7.3 (m, 2H, ArH), 7.4-7.45 (m, 4H, ArH), 7.7-7.75 (m, 4H, ArH).

${ }^{13} \mathrm{C}$ NMR (75 MHz, $\mathrm{CDCl}_{3}$ ): $\delta=107.2$ (2C), 123.7 (4C), 127.3 (2C), 128.7 (4C), 130.7 (2C), 153.3 (2C).

MS (EI, $70 \mathrm{eV}): m / z(\%)=51$ (10), 77 (29), 105 (16), 115 (32), 191 (20), $220(100)[\mathrm{M}]^{+}, 221(18)[\mathrm{M}+\mathrm{H}]^{+}$.

\section{2,5-Bis(4-methoxyphenyl)furan (3b) ${ }^{27}$}

White solid; yield: $83 \mathrm{mg}(59 \%) ; \mathrm{t}_{\mathrm{r}}=17.5 \mathrm{~min}\left[60^{\circ} \mathrm{C}(3 \mathrm{~min})-\right.$ $\left.15{ }^{\circ} \mathrm{C} / \mathrm{min}-270{ }^{\circ} \mathrm{C}(15 \mathrm{~min})\right] ; \mathrm{Mp}=189-192{ }^{\circ} \mathrm{C}$ (hexane); $\mathrm{R}_{\mathrm{f}}=$ 0.20 (Hexane-EtOAc, 4:1).

IR (ATR): 2839, 1600, 1509, $1018 \mathrm{~cm}^{-1}$.

${ }^{1} \mathrm{H} \mathrm{NMR}\left(300 \mathrm{MHz}, \mathrm{CDCl}_{3}\right.$ ): $\delta=3.85$ (s, $6 \mathrm{H}, \mathrm{CH}_{3}$ x 2), 6.58 (s, $2 \mathrm{H}, \mathrm{CH} \times 2$ ), 6.9-6.95 (d, $J=9.0 \mathrm{~Hz}, 4 \mathrm{H}, \mathrm{ArH}), 7.65-7.7$ (d, $J=$ $9.0 \mathrm{~Hz}, 4 \mathrm{H}, \mathrm{ArH})$.

${ }^{13} \mathrm{C}$ NMR (75 MHz, $\left.\mathrm{CDCl}_{3}\right): \delta=55.3$ (2C), 105.5 (2C), 114.1 (4C), 124.0 (2C), 125.0 (4C), 152.8 (2C), 158.8 (2C). 
MS (EI, $70 \mathrm{eV}): m / z(\%)=140$ (14), 265 (87), 266 (16), 280 (100) $[\mathrm{M}]^{+}, 281(20)[\mathrm{M}+\mathrm{H}]^{+}$.

\section{2,5-Di-p-tolylfuran (3c) ${ }^{27}$}

White solid; yield: $112 \mathrm{mg}(90 \%) ; \mathrm{t}_{\mathrm{r}}=17.9 \mathrm{~min}\left[60^{\circ} \mathrm{C}\right.$ (3min)$\left.15{ }^{\circ} \mathrm{C} / \mathrm{min}-270{ }^{\circ} \mathrm{C}(15 \mathrm{~min})\right] ; \mathrm{Mp}=114-117^{\circ} \mathrm{C}$ (hexane); $\mathrm{R}_{\mathrm{f}}=$ 0.73 (Hexane-EtOAc, 4:1).

IR (ATR): 3020, 2914, 2856, 1605, 1503, $1021 \mathrm{~cm}^{-1}$.

${ }^{1} \mathrm{H}$ NMR (300MHz, $\mathrm{CDCl}_{3}$ ): $\delta=2.4\left(\mathrm{~s}, 6 \mathrm{H}, \mathrm{CH}_{3}\right.$ x 2), 6.7 (s, $2 \mathrm{H}, \mathrm{CH} \times 2$ ), 7.2-7.25 (d, $J=8.1 \mathrm{~Hz}, 4 \mathrm{H}, \mathrm{ArH}), 7.6-7.65$ (d, $J=$ $8.1 \mathrm{~Hz}, 4 \mathrm{H}, \mathrm{ArH})$.

${ }^{13} \mathrm{C} \mathrm{NMR}\left(75 \mathrm{MHz}, \mathrm{CDCl}_{3}\right.$ ): $\delta=21.2$ (2C), 106.3 (2C), 123.4 (4C), 128.0 (2C), 129.2 (4C), 136.9 (2C), 153.0 (2C).

MS (EI, $70 \mathrm{eV}): m / z(\%)=129$ (9), 248 (10) [M] $]^{+}, 249$ (20)[M $+\mathrm{H}]^{+}$.

\section{2,5-Bis(4-(trifluoromethyl)phenyl)furan (3d) ${ }^{21}$}

Pale yellow solid; yield: $176 \mathrm{mg}(99 \%) ; \mathrm{t}_{\mathrm{r}}=15.5 \mathrm{~min}\left[60^{\circ} \mathrm{C}(3\right.$ min)- $15{ }^{\circ} \mathrm{C} / \mathrm{min}-270{ }^{\circ} \mathrm{C}(15 \mathrm{~min}) ; \mathrm{Mp}=139-141{ }^{\circ} \mathrm{C}$ (hexane); $\mathrm{R}_{\mathrm{f}}=0.57$ (Hexane-EtOAc, 4:1).

IR (film): $1617 \mathrm{~cm}^{-1}$.

${ }^{1} \mathrm{H}$ NMR (300MHz, $\mathrm{CDCl}_{3}$ ): $\delta=6.9$ (s, 2H, CH x 2), 7.65-7.7 (d, $J=8.1 \mathrm{~Hz}, 4 \mathrm{H}, \mathrm{ArH}), 7.85-7.9$ (d, $J=8.1 \mathrm{~Hz}, 4 \mathrm{H}, \mathrm{ArH})$.

${ }^{13} \mathrm{C}$ NMR (75 MHz, $\mathrm{CDCl}_{3}$ ): $\delta=109.4$ (2C), 123.9 (4C), 124.1 $\left(2 \mathrm{C}, \mathrm{q}, J_{(\mathrm{C}-\mathrm{F})}=271.8 \mathrm{~Hz}\right), 125.8\left(4 \mathrm{C}, \mathrm{q}, J_{(C-F)}=3.7 \mathrm{~Hz}\right), 129.4$ (2C, q, $\left.{ }^{2} J_{(C-F)}=33.2 \mathrm{~Hz}\right), 133.4$ (2C), 152.8 (2C).

MS (EI, $70 \mathrm{eV}): m / z(\%)=145$ (17), 173 (11), 183 (21), 337 (10), 356 (100) $[\mathrm{M}]^{+}, 357(20)[\mathrm{M}+\mathrm{H}]^{+}$.

General procedure for descarboxylative coupling reaction of phenylpropiolic acid.

To a stirred solution of phenylpropiolic acid (4a, $0.6 \mathrm{mmol}$ ) were added $\mathrm{CuO}-\mathrm{Fe}_{3} \mathrm{O}_{4}(50 \mathrm{mg}, 2.1 \mathrm{~mol} \%)$ or $\mathrm{NiO} / \mathrm{Cu}-\mathrm{Fe}_{3} \mathrm{O}_{4}$ (50 mg, 1.5/1.83 mol\%), $\mathrm{Et}_{3} \mathrm{~N}(0.07 \mathrm{~mL}, 138 \mathrm{~mol} \%)$ in DMF $(1.5 \mathrm{~mL})$ under air atmosphere. The corresponding mixture was stirred at $130{ }^{\circ} \mathrm{C}$ during 2 days. The catalyst was removed by a magnet and washed with EtOAc $(2 \times 5 \mathrm{~mL})$ and $\mathrm{H}_{2} \mathrm{O}(2 \times 5$ $\mathrm{mL}$ ). The collected organic phases were dried over $\mathrm{MgSO}_{4}$, and the solvent was removed under reduced pressure. The product was purified by column chromatography to give the corresponding pure product $\mathbf{2 a}$. Yields are included in Scheme 1.

\section{Acknowledgment}

This work was supported by the Spanish Ministerio de Economía y Competitividad (Consolider Ingenio 2010 CSD2007-00006, CTQ2011-24151) and the Generalitat Valenciana (Prometeo 2009/03, FEDER). J. M. P. and R. C. thank the M. E. C., through the FPI program, and the G. V., through the PROMETEO program, respectively, for the corresponding fellowships. We gratefully acknowledge the polishing of our English by Mrs. Oriana C. Townley.

\section{References}

(1) (a) Modern Acetylene Chemistry; Stang, P. J.; Diederich, F., Eds.; VCH: Weinheim, 1995. (b) Brandsma, L. Synthesis of Acetylenes, Allenes and Cumulenes: Methods and Techniques; Elsevier Academic Press: Amsterdam, 2004. (c) Acetylene Chemistry; Diederich, F.; Stang, P. J.; Tykwinski, R. R., Eds.; Wiley-VCH: Weinheim, 2005.

(2) Shun, A. L. K. S.; Tykwinski, R. R. Angew. Chem. Int. Ed. 2006, 45, 1034.

(3) (a) Neenan; T. X.; Whitesides, G. M. J. Org. Chem. 1988, 53, 2489. (b) Tour, J. M. Chem. Rev. 1996, 96, 537. (c) Bunz, U. H. F.; Rubin, Y.; Tobe, Y. Chem. Soc. Rev. 1999, 28, 107.

(4) (a) Ladika, M.; Fisk, T. E.; Wu, W. W.; Jons, S. D. J. Am. Chem. Soc. 1994, 116, 12093. (b) Ohkita, M.; Ando, K.; Suzuki, T.; Tsuji, T. J. Org. Chem. 2000, 65, 4385. (c) Marsden, J. A.; Haley M. M. J. Org. Chem. 2005, 70, 10213.

(5) Crowley, J. D.; Goldup, S. M.; Lee, A.-L.; Leigh, D. A.; McBurney, R. T. Chem. Soc. Rev. 2009, 38, 1530.

(6) For reviews see: (a) Siemsen, P.; Livingston, C.; Diederich, F. Angew. Chem. Int. Ed. 2000, 39, 2632. (b) Stefani, H. A.; Guarezemini, A. S.; Cella, R. Tetrahedron 2010, 66, 7871. (c) Alonso, F.; Yus, M. ACS Catal. 2012, 2, 1441.

(7) (a) Glaser, C. Ber. Dtsch. Chem. Ges. 1869, 2, 422. (b) Hay, A. S. J. Org. Chem. 1962, 27, 3320.

(8) For recent references see: (a) Yadav, J. S.; Reddy, B. V. S.; Reddy, K. B.; Gayathri, K. U.; Prasad, A. R. Tetrahedron Lett. 2003, 44, 6493. (b) Lu, X.; Zhang, Y.; Luo, C.; Wang, Y. Synth. Commun. 2006, 36, 2503. (c) Jiang, H.-F.; Tang, J.-Y.; Wang, A.-Z.; Deng, G.-H.; Yang, S.-R. Synthesis 2006, 1155. (d) Kumar, V.; Chipeleme, A.; Chibale, K. Eur. J. Org. Chem. 2008, 43. (e) Yin, K.; Li, C.; Li, J.; Jia, X. Green Chem. 2011, 13, 591. (f) Zhang, S.; Liu, X.; Wang, T. Adv. Synth. Catal. 2011, 353, 1463. (g) Schmidt, R.; Thorwirth, R.; Szuppa, T.; Stolle, A.; Ondruschka, B.; Hopf, H. Chem. Eur. J. 2011, 17, 8129.

(9) (a) Toda, F.; Tokumaru, Y. Chem. Lett. 1990, 987. (b) Nador, F.; Fortunato, L.; Moglie, Y.; Vitale, C.; Radivoy, G. Synthesis 2009, 4027. (c) Wang, D.; Li, J.; Li, N.; Gao, T.; Hou, S.; Chen, B. Green Chem. 2010, 12, 45.

(10) (a) Auer, S. M.; Schneider, M.; Baiker, A. J. Chem. Soc., Chem. Commun. 1995, 2057. (b) Auer, S. M.; Wandeler, R.; Göbel, U.; Baiker, A. J. Catal. 1997, 169, 1. (c) Zhu, B. C.; Jiang, X. Z. Appl. Organometal. Chem. 2007, 21, 345.

(11) Sharifi, A.; Mirzaei, M.; Naimi-Jamal, M. R. Monatsh. Chem. 2006, 137, 213.

(12) Kuhn, P., Alix, A.; Kumarraja, M.; Louis, B.; Pale, P.; Sommer, J. Eur. J. Org. Chem. 2009, 423.

(13) (a) Oishi, T.; Katayama, T.; Yamaguchi, K.; Mizuno, N. Chem. Eur. J. 2009, 15, 7539. (b) Alonso, F.; Melkonian, T.; Moglie, Y.; Yus, M. Eur. J. Org. Chem. 2011, 2524

(14) Kamata, K.; Yamaguchi, S.; Kotani, M.; Yamaguchi, K.; Mizuni, N. Angew. Chem. Int. Ed. 2008, 47, 2407.

(15) Oishi, T.; Yamaguchi, K.; Mizuno, N. ACS Catal. 2011, $1,1351$.

(16) Xiao, R.; Yao, R.; Cai, M. Eur. J. Org. Chem. 2012, 4178.

(17) Meng, X.; Li, C.; Han, B.; Wang, T.; Chen, B. Tetrahedron 2010, 66, 4029 .

(18) (a) Cano, R.; Ramón, D. J.; Yus, M. Tetrahedron 2011, 67, 5432. (b) Cano, R.; Ramón, D. J.; Yus, M. J. Org. Chem. 2011, 76, 5547. (c) Cano, R.; Yus, M.; Ramón, D. 
J. Tetrahedron 2011, 67, 8079. (d) Cano, R.; Yus, M.; Ramón, D. J. ACS Catal. 2012, 2, 1070. (e) Cano, R.; Yus, M.; Ramón, D. J. Chem. Commun. 2012, 48, 7628.

(19) (a) Martínez, R.; Ramón, D. J.; Yus, M. Adv. Synth.

Catal. 2008, 350, 1235. (b) Martínez, R.; Ramón, D. J.; Yus, M. Org. Biomol. Chem. 2009, 7, 2176. (c) Cano, R.; Yus, M.; Ramón, D. J. Synlett 2011, 2017.

(20) (a) Aliaga, M. J.; Ramón, D. J.; Yus, M. Org. Biomol. Chem. 2010, 8, 43. (b) Cano, R.; Ramón, D. J.; Yus, M. J.Org. Chem. 2010, 75, 3458. (c) Cano, R.; Yus, M.; Ramón, D. J. Tetrahedron 2012, 68, 1393. (d) Pérez, J. M.; Cano, R.; Yus, M.; Ramón, D. J. Eur. J. Org. Chem. 2012, 4548.

(21) Jiang, H.; Zeng, W.; Li, Y.; Wu, W.; Huang, L.; Fu, W. J. Org. Chem. 2012, 77, 5179.

(22) Yu, M.; Pan, D.; Jia, W.; Chen, W.; Jiao, N. Tetrahedron Lett. 2010, 51, 1287.

(23) Feng, X.; Zhao, Z.; Yang, F.; Jin, T.; Ma, Y.; Bao, M. J. Org. Chem. 2011, 696, 1479.

(24) Kude, K.; Hayase, S.; Kawatsura, M.; Itoh, T. Heteroatom Chem. 2011, 22, 397.

(25) Mizuno, N.; Kamata, K.; Nagakawa, Y.; Oishi, T.; Yamaguchi, K. Catal. Today, 2010, 157, 359.

(26) Cheng, T.-P.; Liao, B.-S.; Liu, Y.-H.; Peng, S.-M.; Liu, S.-T. Dalton Trans. 2012, 41, 3468.

(27) Nun, P.; Dupuy, S.; Gaillard, S.; Poater, A.; Cavallo, L.; Nolan, S. P. Catal. Sci. Technol. 2011, 1, 58. 
Please place the graphical abstract and short title of the article here. The short title will be used as a running header.

Homocoupling of terminal alkynes catalyzed by $\mathrm{CuO}-\mathrm{Fe}_{3} \mathrm{O}_{4}$

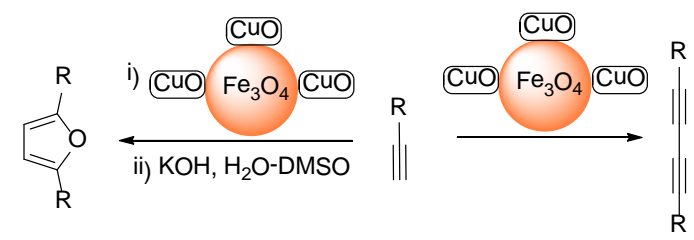

\section{Manuscript submission checklist}

- Statement of significance of work.

In this work a new protocol is introduced to perform the homocoupling of terminal alkynes. The new protocol presents some advantages:

1. The protocol shows a broad scope

2. No using of oxygen pressure or harsh conditions

3. A Solvent-free protocol

4. The lowest copper loading for a heterogeneous catalyst

5. The catalyst is easily removed from the reaction media.

- Full mailing address, telephone, and fax numbers and e-mail address of the corresponding author.

Diego J. Ramón

Dpto. Química Orgánica

Facultad de Ciencias

Universidad de Alicante

Apdo. 99

E-03080-Alicante

Spain

Telephone: (+34) 965903986

Fax: (+34) 965903549

e-mail: djramon@ua.es

- Graphical abstract.

- 5 key words.

- Original Word file.

- Word file saved as a PDF file.

- Original graphics files.

Send the PDF file, statement of significance of work, and full mailing address, telephone, and fax numbers and e-mail address of the corresponding author to the appropriate Regional Editor. Keep the original Word and graphics files for revisions and for final submission after acceptance. 